\title{
Early-onset glaucoma in Axenfeld-Rieger anomaly: long-term surgical results and visual outcome
}

\begin{abstract}
Purpose To determine the long-term surgical and visual outcomes in Indian children with early-onset glaucoma associated with Axenfeld-Rieger anomaly (ARA).

Methods This is a retrospective analysis of 44 eyes of 24 consecutive children with early-onset glaucoma (within 3 years of age) and ARA who underwent glaucoma surgery over a 20-year period (1991-2010) by a single surgeon. Main outcome measures were pre- and postoperative intraocular pressures (IOPs), corneal clarity, visual acuities (VAs), refractive errors, success rate, time of surgical failure, and complications.

Results The series consisted of 38 primary combined trabeculotomy-trabeculectomy (CTT) and 6 primary trabeculectomy procedures (Schlemm's canal could not be identified in these eyes). There was a statistically significant reduction in IOP postoperatively $(27.07 \pm 4.88 v s$ $14.88 \pm 3.62 \mathrm{~mm} \mathrm{Hg} ; P<0.0001)$ with a mean reduction of $45.14 \%$. Success probability by Kaplan-Meier survival analysis was $\mathbf{9 3} \%$ till 5 years, and then $88.1 \%, 82.3 \%, 70.5 \%, 56.4 \%$, and $42.3 \%$ at year $6,7,8,9$, and 10 , respectively. Preoperative corneal edema was present in $43 / 44$ eyes $(97.72 \%)$ and cleared in 42 eyes $(97.67 \%)$. There was one case each with intraoperative hyphema and with shallow chamber postoperatively and both were successfully managed successfully. There was no incidence of endophthalmitis or any other sight-threatening complication. Data on VA were available in 34 eyes (77.3\%). At final follow-up visit, 15 (44.1\%) eyes had best corrected VA $\geqslant 6 / 18$.

Conclusions Primary CTT is safe and effective for early-onset glaucoma associated with ARA. It leads to excellent IOP control and satisfactory visual outcome.
\end{abstract}

'Jasti V Ramanamma Children's Eye Care Centre, Kallam Anji Reddy Campus, LV Prasad Eye Institute, Hyderabad, India

${ }^{2}$ The David Brown Children's Eye Care Centre, Kode Venkatadri Chowdary Campus, Vijayawada, India

Correspondence: AK Mandal, Jasti V Ramanamma Children's Eye Care Centre, LV Prasad Eye Institute, LV Prasad Marg, Banjara Hills, Hyderabad, Telangana 500034, India Tel: +91 40 30612617; Fax: +914023548271. E-mail: mandal@|vpei.org

Received: 29 August 2015 Accepted in revised form: 14 February 2016 Published online: 8 April 2016

Presented in part at the American Academy of Ophthalmology Annual meeting, October 2004, New Orleans, LA, USA.
Eye (2016) 30, 936-942; doi:10.1038/eye.2016.66; published online 8 April 2016
AK Mandal ${ }^{1}$ and $N$ Pehere ${ }^{2}$

\section{Introduction}

Axenfeld-Rieger anomaly (ARA) is the result of abnormal migration of neural crest cells and encompasses a spectrum of disorders that include a prominent and anteriorly displaced Schwalbe's line, iridocorneal adhesions, iris anomalies like ectropion uveae, atrophic iris holes, and so on. Slightly more than half of the patients with ARA develop glaucoma. ${ }^{1}$ This may commonly become manifest in childhood or young adulthood, but it may appear at any age. ${ }^{2,3}$ It may be present in infancy as buphthalmos or as congenital corneal edema. ${ }^{3}$

Secondary developmental glaucoma in childhood due to ARA is frequently treated with medications, but medical therapy alone often fails to control the intraocular pressure (IOP) and prevent progression of the disease. When surgical intervention is required, particularly in early-onset glaucoma, controversy exists regarding the procedure of choice. ${ }^{4}$ Rice $^{4}$ reported extremely poor results and commented that goniotomy is probably contraindicated in Axenfeld's syndrome. In many cases, iridocorneal attachments interfere with goniotomy. ${ }^{1,5}$ In addition, goniotomy has the disadvantage that it requires the presence of clear cornea to afford an adequate intraoperative view of the angle. ${ }^{6-8}$ Walton $^{5}$ reported success with goniotomy in two of five patients with iridocorneal dysgenesis with a normal pupil. Different forms of congenital glaucoma do vary in their response to goniotomy. Alternative angle surgery, that is, ab externo traebeculotomy, has also been reported ${ }^{9}$ to be effective in the management of glaucoma in iridocorneal dysgenesis. Based on the histopathological features of ARA, Shields ${ }^{2}$ suggested that the angle surgery usually would not be successful in eyes with early-onset glaucoma in ARA. He advocated trabeculectomy possibly with 
antimetabolites in this situation. ${ }^{1}$ Mandal et al ${ }^{10-18}$ reported combined trabeculotomy-trabeculectomy (CTT) as a successful surgical procedure in primary developmental glaucoma as well as early-onset glaucoma in Sturge-Weber syndrome. The aim of the present study was to evaluate our surgical results in early-onset glaucoma in ARA.

\section{Patients and methods}

We reviewed medical records of patients with ARA with early-onset glaucoma who underwent glaucoma surgery between 1991 and 2010 by a single surgeon (AKM) at the LV Prasad Eye Institute (LVPEI), Hyderabad, India. For the purpose of this study, early-onset glaucoma was defined as glaucoma that occurred either at birth or at any point of time within 3 years of life (congenital and infantile glaucoma). Patients with history of previous glaucoma surgery were excluded. A total of 24 patients (44 eyes) were included. CTT was used as the primary therapeutic modality. Given that Schlemm's canal could not be identified, four patients (six eyes) could not undergo CTT and these eyes underwent trabeculectomy alone. These four patients were excluded from analysis of cumulative success probability.

Ethical approval was obtained from the Ethics committee for Human Research of the LVPEI and the research adhered to the tenets of the Declaration of Helsinki. Informed consent was obtained from the parents of all children. The following information was collected for each patient: demographic details, age at presentation, age at surgery, pre- and postoperative corneal clarity, diameter, pre- and postoperative IOP, visual acuity (VA), refractive error, number of antiglaucoma medications, bleb characteristics, any complications, duration of follow-up, and systemic features, if any.

\section{Surgical procedure}

The surgical technique employed in all cases was primary CTT. This technique has been previously described by us. ${ }^{16-19}$ In brief, the Schlemm's canal was dissected under a partial thickness limbal-based triangular scleral flap and trabeculotomy ab externo was performed on both sides of the radial incision. Trabeculectomy was then performed in the usual manner. In cases of bilateral affliction, after completion of surgery on the first eye, the second eye was operated using a similar technique but with a new set of instruments, drapes, gown, gloves, and so on, simulating a surgical procedure on a different patient. None of the patients received any antimetabolite intraoperatively in the primary surgery. However, it was considered during repeat surgery.

\section{Postoperative regimen}

All patients were treated with topical corticosteroids (prednisolone acetate 1\% 6 times/day tapered over 6 weeks), cycloplegic (cyclopentolate 1\% 3 times a day for 3 weeks), and topical antibiotic (4 times/day for 1 week). All patients were examined on postoperative day 1 , weeks 1 and 3 in the office followed by examination under anesthesia at week 6 , and every 3 months thereafter. At each visit, patients were examined under surgical microscope, slit-lamp biomicroscope for the anterior chamber depth, corneal clarity, horizontal corneal diameter, IOP, bleb appearance, and examination fundus. Refraction was done and visual acuity was measured wherever possible.

\section{Success criteria}

Surgical success and failure were defined before data analysis. The surgery was considered a complete success when the IOP (without antiglaucoma medication) was $<16 \mathrm{~mm} \mathrm{Hg}$ in patients examined under general anesthesia with Perkin's hand-held applanation tonometer or $<21 \mathrm{~mm} \mathrm{Hg}$ in patients who were old enough to be examined with the slit-lamp (IOP measured with Goldmann Applanation tonometer under topical anesthesia). Qualified success was defined when such IOP was maintained with one antiglaucoma medication. Failure was defined when such IOP could not be achieved even with the addition of one antiglaucoma medications or there was persistent corneal edema or sight-threatening complications or the need for repeat surgery. In addition, repeat surgery was considered as failure.

\section{Statistical analysis}

Results are expressed as mean \pm SD. A $P$-value of $<0.05$ was considered statistically significant. Paired $t$-test was used to compare the pre and postoperative IOP. KaplanMeier survival analysis was done to calculate the cumulative success probability of the surgery using the SPSS (SPSS for Windows, version 16.0, SPSS, Chicago, IL, USA) software.

\section{Results}

\section{Demographic data}

Twenty-four patients (44 eyes) were included in the study. Patient characteristics are summarized in Table 1. The median age at surgery was 2.5 months (range 20 days -3 years). The study population primarily had congenital and infantile-onset glaucoma. There were 15 males and 9 females. Four patients (16.7\%) had unilateral affliction, whereas 20 (83.3\%) had bilateral affliction. 
Table 1 Sociodemographic data of 44 eyes of 24 patients with Axenfeld-Rieger anomaly

\begin{tabular}{|c|c|}
\hline Demographic & No. $(\%)$ \\
\hline \multicolumn{2}{|c|}{ Age at surgery (months) } \\
\hline Mean \pm SD & $4.27 \pm 7.18$ \\
\hline Range & 20 days -3 years \\
\hline \multicolumn{2}{|l|}{ Gender } \\
\hline Male & $15(62)$ \\
\hline Female & $9(38)$ \\
\hline \multicolumn{2}{|l|}{ Affliction } \\
\hline Unilateral & $4(17)$ \\
\hline Bilateral & $20(83)$ \\
\hline \multicolumn{2}{|c|}{ Horizontal corneal diameter at presentation $(\mathrm{mm})$} \\
\hline Mean \pm SD & $12.44 \pm 0.96$ \\
\hline Range & $10-14.5$ \\
\hline \multicolumn{2}{|c|}{ Corneal clarity at presentation } \\
\hline Clear & $1(2)$ \\
\hline Edema & $43(98)$ \\
\hline \multicolumn{2}{|c|}{ Corneal clarity at last visit } \\
\hline Clear & $43(98)$ \\
\hline Edema & $1(2)$ \\
\hline \multicolumn{2}{|c|}{ Preoperative IOP $(\mathrm{mm} \mathrm{Hg})$} \\
\hline Mean \pm SD & $27.1 \pm 4.9$ \\
\hline Range & $16-32$ \\
\hline \multicolumn{2}{|c|}{ Postoperative IOP $(\mathrm{mm} \mathrm{Hg})^{\mathrm{a}}$} \\
\hline Mean \pm SD & $14.9 \pm 3.6$ \\
\hline Range & $9-24$ \\
\hline \multicolumn{2}{|c|}{ Follow-up (months) } \\
\hline Mean \pm SD & $65.2 \pm 50.1$ \\
\hline Range & $6-153$ \\
\hline
\end{tabular}

$\overline{{ }^{a} \text { Intraocular pressure (IOP) recorded at the last follow-up visit; } P<0.0001}$.

The mean follow-up was $65.19 \pm 50.10$ months (range 6-153 months, median 66 months). Seven eyes of four patients with bilateral affliction had associated iris changes in the form of ectropion uveae, atrophic iris holes, and correctopia. All the patients had posterior embryotoxon and five patients with bilateral affliction had systemic features in the form of maxillary hypoplasia.

\section{Intraocular pressure}

All the patients were using one topical antiglaucoma combination therapy containing dorzolamide hydrochloride and timolol maleate. The mean preoperative IOP was $27.07 \pm 4.88 \mathrm{~mm} \mathrm{Hg}$ (range 16-32 $\mathrm{mm} \mathrm{Hg}$, 95\% CI 25.57-28.57). Although the lower range of IOP was $16 \mathrm{~mm} \mathrm{Hg}$ preoperatively in some patients, we decided to operate upon them because these patients had associated corneal edema and megalocornea. At the final follow-up visit, the mean IOP was significantly lower than the preoperative level $14.88 \pm 3.62 \mathrm{mmHg}$ (range 9-24 $\mathrm{mm} \mathrm{Hg}$,
95\% CI 13.85-16.09, $P<0.0001$, paired $t$-test) with the percentage reduction being 45.14. At last visit postoperatively, 1-2 antiglaucoma medications had to be used in $12 / 44$ eyes (27.27\%).

\section{Corneal clarity}

Preoperative corneal edema was present in 43/44 eyes $(97.72 \%)$ that cleared in 42 of them $(97.67 \%)$. One eye had persistent corneal edema. Figures $1 \mathrm{a}$ and $\mathrm{b}$ show the preoperative and 1-year postoperative appearance of the right cornea in a child who underwent surgery at the age of 1 month. Similarly, Figures $1 \mathrm{c}$ and d show the preoperative and 1-year postoperative appearance of the left cornea of the same child. Figures $1 \mathrm{e}$ and $\mathrm{f}$ show the 1-year postoperative bleb appearance of the right and left eyes respectively.

\section{Refractive error}

Data about refractive error were available for all eyes. The majority of the eyes $(38,86 \%)$ had some form of astigmatism. Of these, 12 (32\%) had compound hyperopic astigmatism, 11 (29\%) had mixed astigmatism, 10 (26\%) had compound myopic astigmatism, and 5 (13\%) had simple myopic astigmatism. The remaining eyes had simple myopia $(1,2 \%)$ and simple hyperopia $(5,11 \%)$.

\section{Visual acuity}

At the final postoperative visit, reliable best corrected visual acuity (BCVA) assessment by Snellen chart was available in 34 eyes (77.3\%). Of these, 15 (44.1\%) had $\mathrm{BCVA} \geqslant 6 / 18$ in the affected eye and $10(29.4 \%)$ had BCVA $<6 / 18-3 / 60$ (low vision (LV)). Nine eyes (26.47\%) had BCVA $<3 / 60$ (blind). However, when we classified our visual outcome based on the World Health Organization criteria of visual loss (BCVA in the better eye) for 19 patients (34 eyes; 15 bilateral and 4 unilateral), 11 patients $(58 \%)$ had BCVA $\geqslant 6 / 18$ (and were considered normal), $7(37 \%)$ had LV, and 1 (5\%) was blind.

\section{Success rate}

Complete success as defined in the present study was obtained in 30 eyes (68.2\%). Qualified success was obtained in 9 eyes (20.4\%). Five eyes (11.4\%) were classified as failures. Kaplan-Meier survival analysis revealed success probability of $93 \%$ till 5 years and then it was $88.1 \%, 82.3 \%, 70.5 \%, 56.4 \%$, and $42.3 \%$ at year $6,7,8$, 9 , and 10, respectively. Figure $2 \mathrm{a}$ represents the cumulative success probability for eyes with complete success and Figure $2 \mathrm{~b}$ represents the cumulative success 

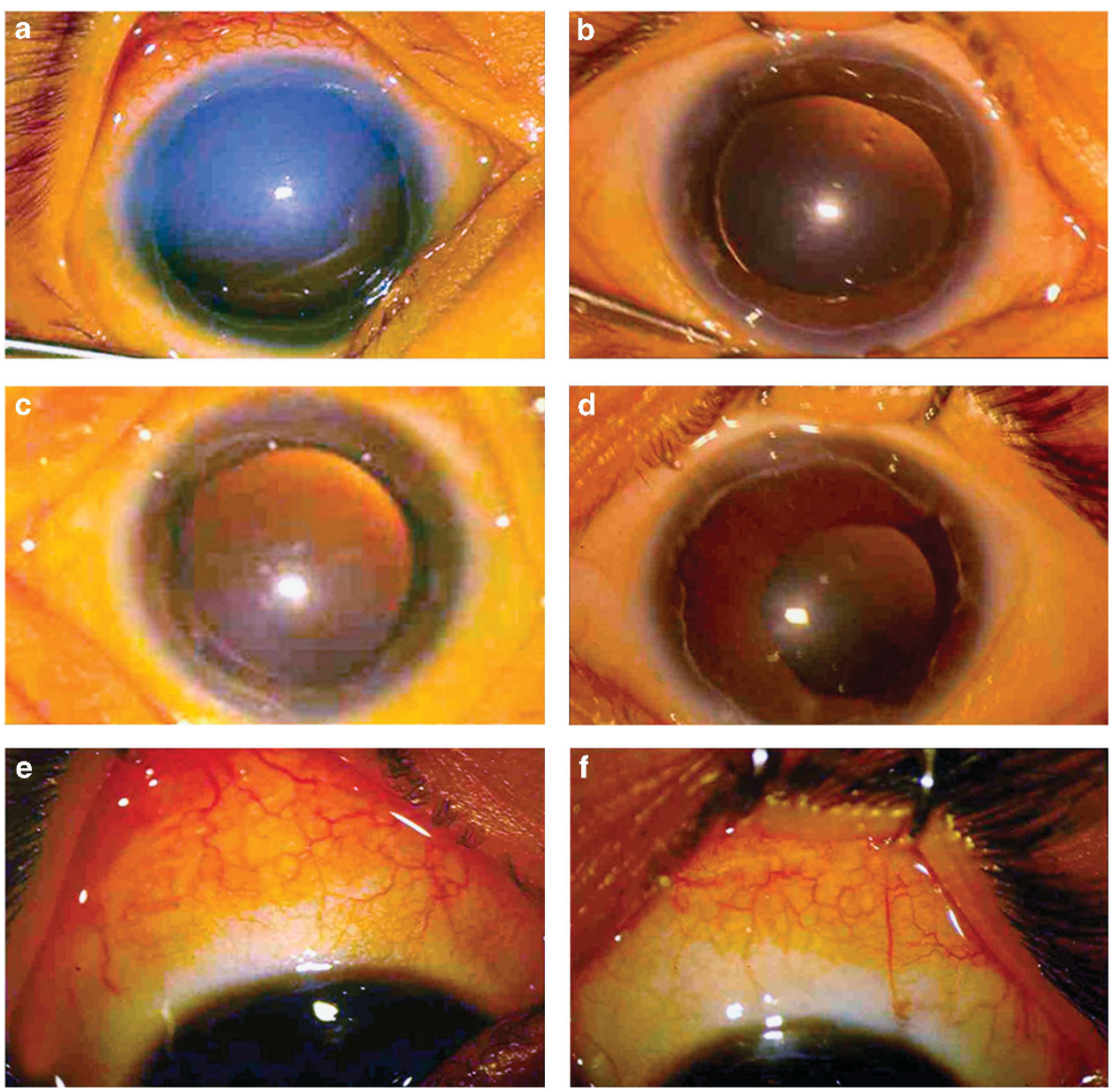

Figure 1 (a) Preoperative appearance of the right eye showing corneal edema in a 1-month-old child with Axenfeld-Rieger anomaly. (b) The 1-year postoperative appearance of the eye seen in (a) showing clear cornea. (c) Preoperative appearance of the left eye of the same child showing corneal edema. (d) The 1-year postoperative appearance of the eye seen in (c) showing clear cornea. (e) The 1-year postoperative appearance of the bleb of the right eye. (f) The 1-year postoperative appearance of the bleb of the left eye.

probability for eyes with complete plus qualified success.

\section{Surgical complications}

Intraoperative hyphema occurred in one eye that was well managed. Postoperative persistent shallow anterior chamber was seen in one eye that was surgically reformed with air bubble injection into the anterior chamber on postoperative day 3 .

\section{Repeat surgery}

None of the patients required any postoperative manipulation for suture release or needling. Repeat trabeculectomy with mitomycin- $C$ was performed in one eye for uncontrolled IOP even with three antiglaucoma medications. Transscleral cyclophotocoagulation was done in one eye of a patient with advanced disc damage and poor visual potential.

\section{Discussion}

Glaucoma associated with the ARA is believed to be the result of either compression of the trabecular meshwork or the incomplete development of the meshwork or Schlemm's canal. The mechanism of early-onset glaucoma in ARA is developmental arrest that accounts for high insertion of anterior uvea into the posterior trabecular meshwork, similar to the alternative seen in primary congenital glaucoma. It is basically a surgical disease.

The classic operation for the treatment of primary infantile glaucoma is Barkan's goniotomy. Analysis of the results of treatment of simple congenital glaucoma reveals that goniotomy is very effective and safe procedure and 
a

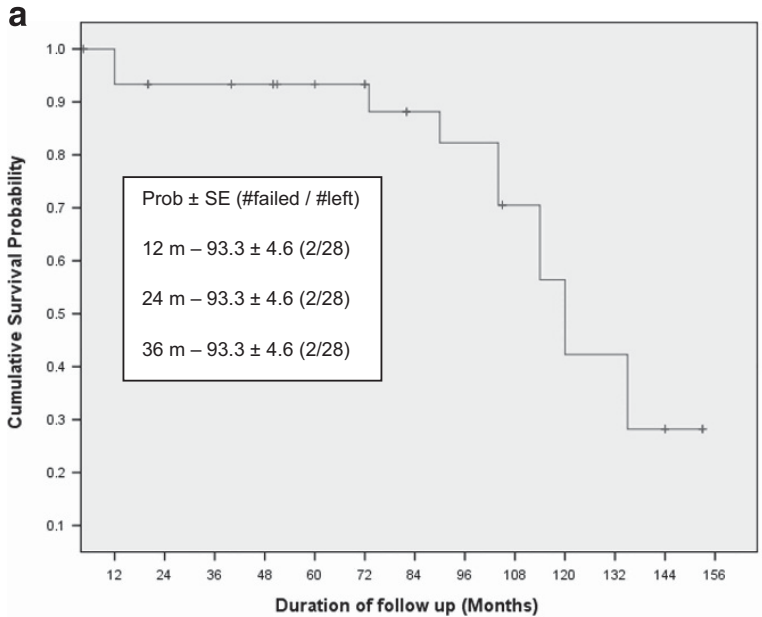

b

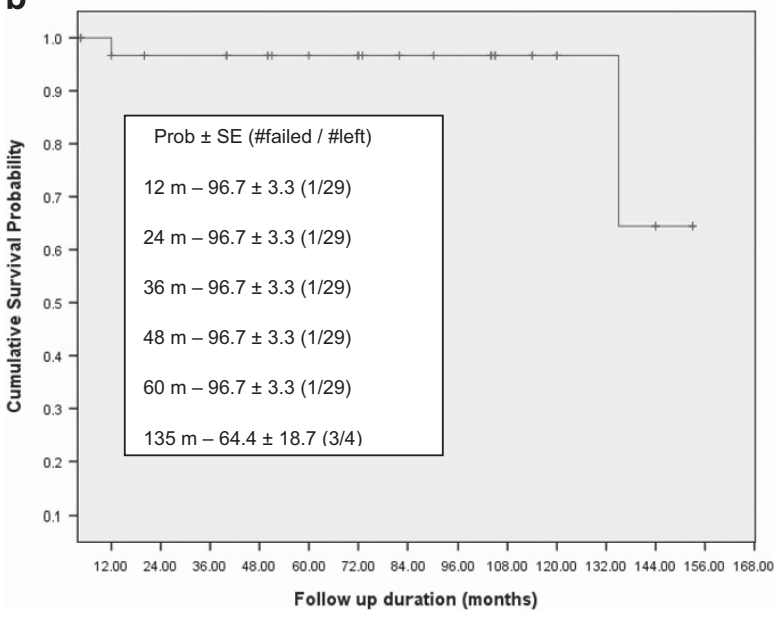

Figure 2 (a) Kaplan-Meier survival curve showing complete success for IOP control in children with Axenfeld-Rieger anomaly. (b) Kaplan-Meier survival curve showing complete plus qualified success for IOP control in children with AxenfeldRieger anomaly.

can be expected to control the glaucoma in $\sim 85 \%$ of the eyes. However, different forms of congenital glaucoma do vary in their response to goniotomy or other forms of surgery.

Rice $^{4}$ reported extremely poor results of goniotomy in Axenfeld syndrome. Out of 19 eyes of 11 cases treated by goniotomy, the glaucoma was controlled in only two eyes. Based on these results, Rice ${ }^{4}$ commented that goniotomy is probably contraindicated in Axenfeld syndrome. Several other authors also reported poor results of goniotomy in ARA. Wallace et al ${ }^{19}$ performed five gonitomies with no success in patients with anterior segment dysgenesis. Walton ${ }^{5}$ reported success with goniotomy in two of five patients with iridocorneal dysgenesis with a normal pupil. Shields $e t$ al ${ }^{1,2}$ and Walton ${ }^{5}$ reported the technical problem of interference because of iridocorneal attachments during goniotomy in ARA. The other prerequisite for successful goniotomy is clear cornea in order to have a good intraoperative view of the angle. In the present series, 43 out of 44 (98\%) eyes had significant corneal edema; in these cases, goniotomy would have been impossible.

Trabeculotomy ab externo may be considered as an alternative surgical technique in the presence of corneal edema. The advantages of trabeculotomy ab externo over the alternative procedure of goniotomy have been reported by Luntz. ${ }^{9}$ Anderson, Quigley, and Shaffer reported that the two procedures were equally effective. ${ }^{18}$ Several successful reports of primary trabeculectomy for developmental glaucoma have been reported in the literature. ${ }^{8}$ Luntz ${ }^{9}$ reported good IOP control in two of six eyes with iridocorneal dysgenesis after trabeculotomy. Shields $\mathrm{et} \mathrm{al}^{1}$ pointed out that the incomplete development of Schlemm's canal and the anterior meshwork precludes success with either goniotomy or trabeculotomy in many cases of ARA and recommended trabeculectomy as the procedure of choice for most patients with glaucoma secondary to ARA. Wallace $e t a l^{19}$ reported a small series of patients with anterior segment dysgenesis; 9 (69\%) of 13 eyes achieved IOP control and stabilization of optic disc appearance after one or more procedure.

Trabeculectomy has its own limitations with low success rate in the younger age group than in older glaucoma patients. The barriers to success of filtering surgery in children include a thick, active Tenon's capsule and rapid wound healing response. Fibrosis is the subTenon's space involving the episcleral; Tenon's and conjunctival interface are the leading causes of filtering surgery failure.

More than one procedure is often required to improve the surgical success, causing inconvenience to the surgeon and patient. Children born with primary congenital glaucoma have poor surgical prognosis. $8,10,15,20$ It is quite natural to assume that the success probability of earlyonset glaucoma in ARA will be even poorer. However, the chances of success are best with the first surgical procedure. $^{20,21}$

The most compelling argument favoring primary CTT in early-onset glaucoma in ARA was the higher incidence of successful IOP control with a single operative procedure as has been already reported by us. ${ }^{12-18}$ The aim of the present study as to evaluate the surgical outcome of infants with secondary glaucoma associated with ARA. Kaplan-Meier survival analysis demonstrated the probability of complete success of $93 \%$ till 5 years, and then $88.1 \%, 82.3 \%, 70.5 \%, 56.4 \%$, and $42.3 \%$ at year $6,7,8$, 9 , and 10, respectively. Our success rate with initial surgery is significantly better than most of the reported results of initial goniotomy and trabeculectomy. In CTT, trabeculotomy takes care of the angle anomaly, whereas 
trabeculectomy provides an alternate outflow pathway. This may be the reason for long-term success of this procedure. ${ }^{10,18}$ We observed diffuse functioning filtering bleb in 30 eyes (68\%). However, the superiority of CTT over trabeculectomy is debatable. Further prospective randomized studies are required to explore this issue. Nonetheless, a comparative study of the two surgical procedures does not seem feasible given the rarity of disease and diversity of presentation to the extent that no two cases are alike.

One of the objectives of the study was to determine the functional outcome of the early-onset glaucoma in ARA. Fifteen eyes $(44.1 \%)$ had BCVA $\geqslant 6 / 18$ at the final follow-up in the affected eye. Ten eyes (29.41\%) had LV $(<6 / 18-3 / 60)$ and 9 eyes $(26.47 \%)$ were blind $(<3 / 60)$. Early detection, prompt surgical correction, and aggressive amblyopia therapy were the key factors for our improved results. The majority of the eyes (86\%) had some form of astigmatism.

There were no serious intraoperative or postoperative complications. Of the minor postoperative complications, shallow anterior chamber occurred in one eye in first postoperative week that required surgical reformation. In this series, 20 patients underwent simultaneous bilateral surgery to avoid another longer anesthesia, despite theoretical risk of endophthalmitis. There was no incidence of bleb leakage, bleb-related infection, or endophthalmitis. In six eyes of three patients, trabeculotomy was attempted but could not be performed successfully and they underwent trabeculectomy alone. The reason for this is the absence of Schlemm's canal or presence of a rudimentary Schlemm's canal in these cases as reported by Shields. ${ }^{2}$

We expect that the outcome of our study will have a positive impact on parental counseling. The benefits of long-term IOP control and good visual outcome with single surgery as achieved in this series is attractive to both parents and treating ophthalmologist. However, possibility of long-term drift of results should be borne in mind as has been reported in literature. ${ }^{4,22,23}$ If primary CTT fails, we normally perform trabeculectomy with mitomycin-C in another quadrant as a secondary procedure. ${ }^{24,25}$ There is frequent need to use antiglaucoma medications in these patients. This underlines the importance of regular lifelong follow-up.

The limitations of this study are its nonrandomized, retrospective design.

In conclusion, primary CTT is a safe and effective procedure for early-onset glaucoma in ARA. The visual outcome was satisfactory in most of the patients. There is need for regular lifelong follow-up of these patients.

\section{Summary}

What was known before

- Early-onset glaucoma in Axenfeld-Rieger anomaly (ARA) is a surgical disease.

- Angle incision surgery, that is, goniotomy and trabeculotomy, has poor success rates.

What this study adds

- Primary combined trabeculotomy-trabeculectomy (CTT) is safe and effective in early-onset glaucoma in ARA.

- Visual outcome is satisfactory.

- Results of primary CTT are helpful in parental counseling and may be considered as the first surgical option in earlyonset glaucoma in ARA.

\section{Conflict of interest}

The authors declare no conflict of interest.

\section{Acknowledgements}

This study was supported in part by the Hyderabad Eye Research Foundation, LV Prasad Eye Institute,

Hyderabad, India.

\section{References}

1 Shields MB, Buckley E, Klintworth GK, Thresher R. Axenfeld-Rieger syndrome. A spectrum of developmental disorders. Surv Ophthalmol 1985; 29: 387-409.

2 Shields MB. Axenfeld-Rieger syndrome: a theory of mechanism and distinctions from the iridocorneal endothelial syndrome. Trans Am Ophthalmol Soc 1983; 81: 736-784.

3 Waring GO III, Rodrigues MM, Laibson PR. Anterior chamber cleavage syndrome. A stepladder classification. Surv Ophthalmol 1975; 20: 3-27.

4 Rice NSC. The surgical management of the congenital glaucomas. Aust J Ophthalmol 1977; 5: 174-179.

5 Walton DS. Iridocorneal dysgenesis with glaucoma. In: Epstein DL (eds) Chandler and Grant's Glaucoma, 3rd ed, Chap 38. Lea \& Febiger: Philadelphia, 1986, pp 501-505.

6 de Luise VP, Anderson DR. Primary infantile glaucoma (congenital glaucoma). Surv Ophthalmol 1983; 28: 1-19.

7 Luntz MH. The advantages of trabeculotomy over goniotomy. J Pediatr Ophthalmol Strabismus 1984; 21: 150-153.

8 O'Connor G. Combined trabeculotomy-trabeculectomy for congenital glaucoma (editorial). Br J Ophthalmol 1994; 78: 735.

9 Luntz MH. Congenital, infantile, and juvenile glaucoma. Ophthalmology 1979; 86: 793-802.

10 Mandal AK. Current concepts in the diagnosis and management of developmental glaucomas. Indian J Ophthalmol 1993; 41: 51-70.

11 Mandal AK. Microsurgical technique combines trabeculotomy and trabeculectomy to treat developmental glaucoma. Ocular Surg News, Internat Ed 1994; 5: 38-43.

12 Mandal AK, Naduvilath TJ, Jayagandhan A. Surgical results of combined trabeculotomy-trabeculectomy for developmental glaucoma. Ophthalmology 1998; 105: 974-982. 
13 Mandal AK. Primary combined trabeculotomytrabeculectomy for early onset glaucoma in Sturge-Weber syndrome. Ophthalmology 1999; 106: 1621-1627.

14 Mandal AK, Bhatia PG, Gothwal VK, Reddy VM, Sriramulu P, Prasad MS. Safety and efficacy of simultaneous bilateral primary combined trabeculotomy-trabeculectomy for developmental glaucoma in India. Indian J Ophthalmol 2002; 50: 13-19.

15 Mandal AK, Gothwal VK, Bagga H, Nutheti R, Mansoori T. Outcome of surgery on infants younger than 1 month of age with congenital glaucoma. Ophthalmology 2003; 110: 1909-1915.

16 Mandal AK, Bhatia PG, Bhaskar A, Nutheti R. Long-term surgical and visual outcomes in Indian children with developmental glaucoma operated within 6 months of birth. Ophthalmology 2004; 111: 283-290.

17 Mandal AK, Matalia JH, Nutheti R, Krishnaiah S. Combined trabeculotomy and trabeculectomy in advanced primary developmental glaucoma with corneal diameter of $14 \mathrm{~mm}$ or more. Eye 2006; 20: 135-143.

18 Mandal AK, Gothwal VK, Nutheti R. Surgical outcome of primary developmental glaucoma: a single surgeon's experience long term experience from a tertiary eye care centre in India. Eye 2007; 21: 764-774.

19 Wallace DK, Plager DA, Snyder SK, Raiesdana, Helveston EM, Ellis FD. Surgical results of secondary glaucomas in childhood. Ophthalmology 1998; 105: 101-111.

20 Hass J. Principles and problems of therapy in congenital glaucoma. Invest Ophthalmol 1968; 7: 140-146.

21 McPherson Jr SD, Berry DP. Goniotomy vs external trabeculotomy for developmental glaucoma. Am J Ophthalmol 1983; 95: 427-431.

22 Dietlein TS, Jacobi PC, Krieglstein GK. Prognosis of ab externo surgery for primary congenital glaucoma. Br J Ophthalmol 1999; 83: 317-322.

23 Russell-Eggitt IM, Rice NS, Jay B, Wyse RK. Relapse following goniotomy for congenital glaucoma due to trabecular dysgenesis. Eye 1992; 6: 197-200.

24 Mandal AK, Walton DS, John T, Jayagandan A. Mitomycin C-augmented trabeculectomy in refractory congenital glaucoma. Ophthalmology 1997; 104: 996-1001.

25 Mandal AK, Prasad K, Naduvilath TJ. Surgical results and complications of mitomycin C-augmented trabeculectomy in refractory developmental glaucoma. Ophthalmic Surg Lasers 1999; 30: 473-480. 